\title{
Weibull stress analysis in local approach to fracture
}

\author{
Yuebing $\mathrm{Li}^{\mathrm{a}, \mathrm{c}}$, Zihang Wang ${ }^{\mathrm{a}}$, Yuebao Lei ${ }^{\mathrm{a}, \mathrm{d}}$, Guian Qian ${ }^{\mathrm{b}, *}$, Mingjue Zhou ${ }^{\mathrm{a}, \mathrm{c}, *}$, Zengliang Gao ${ }^{\mathrm{a}, \mathrm{c}}$, \\ Filippo Berto ${ }^{\mathrm{e}}$ \\ ${ }^{a}$ Institute of Process Equipment and Control Engineering, Zhejiang University of Technology, Hangzhou 310032, China \\ ${ }^{\mathrm{b}}$ State Key Laboratory of Nonlinear Mechanics, Institute of Mechanics, Chinese Academy of Sciences, Beijing 100190, China \\ ${ }^{\mathrm{c}}$ Engineering Research Center of Process Equipment and Re-manufacturing of Ministry of Education, Zhejiang University of Technology, Hangzhou 310032, China \\ ${ }^{\mathrm{d}}$ EDF Energy Nuclear Generation Ltd., Barnett Way, Barnwood, Gloucester GL4 3RS, United Kingdom \\ ${ }^{\mathrm{e}}$ Department of Mechanical and Industrial Engineering, Norwegian University of Science and Technology (NTNU), Richard Birkelands vei 2b, 7491 Trondheim, Norway
}

\section{A R T I C L E I N F O}

\section{Keywords:}

Weibull stress

Local approach

Fracture

$T$-stress

Constraint effect

\begin{abstract}
A B S T R A C T
As a measure of the probability of cleavage fracture, the Weibull stress within the framework of local approach has the potential capability to predict constraint effects on fracture of structural steels. This paper mainly analyzes Weibull stress considering constraint effect using constraint parameters $T$-stress and $Q$. Weibull stress is solved with constraint effects characterized by $T$-stress for elastic material and $Q$ for elastic plastic material. These solutions are verified with existing solutions for $T=0$ and finite element solutions including modified boundary layer models, contact tension models and single-edge bend models. Good agreement has been obtained in all cases. The Weibull stress solutions can be further adopted to predict scale fracture toughness.
\end{abstract}

\section{Introduction}

For ferritic steels at temperatures in the ductile-to-brittle transition region, unstable cleavage fracture is still a key issue in the structural integrity assessment. Local approaches to (cleavage) fracture (LAF) based on micro-mechanical models of failure have been used to predict material failure caused by transgranular cleavage fracture, in which stresses local to the crack tip are related to the critical conditions required for cleavage fracture. Among the available local approach models, the most widely used one is the Beremin model $[1,2]$, which introduced a new parameter, the Weibull stress, defined at the local material volume close to the crack tip to predict the probability of fracture occurrence. The model had been adopted in structural integrity assessment as an alternative approach to demonstrate fracture avoidance [3]. The Weibull stress as a probabilistic fracture driving force is a key parameter in the application of the Beremin model to a crack in a component. Recently, Qian et al. [4] developed a new local approach to fracture based on Beremin model. The new approach is more consistent and predict a more precise fracture probability and is now applied in fracture of reactor pressure vessel [5], fracture of concrete [6] and fatigue assessment [7]. However, unlike the conventional fracture mechanics method, there is no a relationship between the Weibull stress and the applied load for given geometry conditions and material properties. Detailed finite element (FE) analysis is required in general to evaluate the Weibull stress during the fracture assessment. This could be one of the reasons why the Beremin model is not widely used in engineering structural integrity assessment. In fact, the Beremin model parameters can be calibrated using the fracture toughness test results based on the cleavage crack initiation conditions determined from fracture tests. This means that there is a unique relationship between the conventional fracture mechanics parameter and the Weibull stress at the critical conditions describing the on-set of cleavage fracture. It can be inferred that such a relationship between the convectional fracture mechanics parameters, such as stress intensity factor (SIF) or Jintegral, and the Weibull stress exists for given geometry and material properties when the crack tip constraint conditions are also considered. Some efforts have been made to obtain such relationships. Lei et al. [8] has developed analytical and semi-analytical expressions for Weibull stress in terms of SIF or $J$ and material properties under plane strain conditions. These expressions enable Weibull stress to be estimated in structural integrity assessment without the need of detailed FE analyses [9] and provide insight into the use of the Weibull stress as a parameter for the prediction of cleavage failure of cracked bodies. However, for general cases, the Weibull stress should be linked to both fracture mechanics driving force parameters and the crack tip constraint parameters, such as $T$-stress $[10,11]$ for elastic materials and $Q[12,13]$ for

\footnotetext{
* Corresponding authors at: State Key Laboratory of Nonlinear Mechanics, Institute of Mechanics, Chinese Academy of Sciences, Beijing 100190, China (G. Qian). Institute of Process Equipment and Control Engineering, Zhejiang University of Technology, Hangzhou 310032, China (M. Zhou).

E-mail addresses: qianguian@imech.ac.cn (G. Qian), zhoumingjue@zjut.edu.cn (M. Zhou).
} 


\begin{tabular}{|c|c|c|c|}
\hline \multicolumn{2}{|c|}{ Nomenclature } & $W$ & width [mm] \\
\hline$a$ & crack length $[\mathrm{mm}]$ & $\beta$ & constraint parameter $[-]$ \\
\hline$b$ & fracture ligament $[\mathrm{mm}]$ & $\varepsilon$ & strain $[-]$ \\
\hline$B$ & thickness [mm] & $\zeta$ & coefficient for $\Phi[-]$ \\
\hline$E$ & elastic module [MPa] & $\theta$ & crack tip angle [rad] \\
\hline$J$ & $J$-integral $\left[\mathrm{kJ} / \mathrm{m}^{2}\right]$ & $\lambda$ & coefficient to determine fracture process zone $[-]$ \\
\hline K & stress intensity factor $\left[\mathrm{MPa}^{*} \mathrm{~mm}^{0.5}\right]$ & $\xi$ & coefficient for $\Phi[-]$ \\
\hline$m$ & Weibull module $[-]$ & $\rho$ & notch radius $[\mathrm{mm}]$ \\
\hline$n$ & hardening exponent [-] & $\sigma$ & stress $[\mathrm{MPa}]$ \\
\hline$n_{e}$ & number of elements $[-]$ & $\sigma_{\mathrm{w}}$ & Weibull stress $[\mathrm{MPa}]$ \\
\hline$Q$ & constraint parameter $[-]$ & $\left(\sigma_{\mathrm{w}}\right)_{T=0}$ & Weibull stress under $T=0[\mathrm{MPa}]$ \\
\hline$r$ & distance from the crack tip [mm] & $\sigma_{1}$ & maxprincipal stress $[\mathrm{MPa}]$ \\
\hline$R_{1}$ & upper limit of integration [mm] & $\sigma_{0}$ & yield stress $[\mathrm{MPa}]$ \\
\hline$R_{2}$ & lower limit of integration [mm] & $\sigma_{\mathrm{u}}$ & Weibull module $[-]$ \\
\hline$T$-stress & constraint parameter $[\mathrm{MPa}]$ & $\sigma_{\mathrm{yy}}$ & open stress of the crack tip [MPa] \\
\hline$V_{0}$ & reference volume $\left[\mathrm{mm}^{3}\right]$ & $v$ & Poisson's ratio $[-]$ \\
\hline$V_{\mathrm{p}}$ & fracture process zone $\left[\mathrm{mm}^{3}\right]$ & $\Phi$ & $\sigma_{\mathrm{w}} /\left(\sigma_{\mathrm{w}}\right)_{T=0}[-]$ \\
\hline
\end{tabular}

elastic-plastic materials, because the crack tip fields can be described by fracture mechanics parameter and the crack tip constraint parameters. Such relationships are currently unavailable.

In local approaches, the effect of crack tip constraint on the cleavage fracture can be characterized by Weibull stress itself. The local parameter Weibull stress has been used to correlate the fracture toughness values of materials obtained at various constraint levels although there is no general relationship between the local parameter and the global fracture mechanics and constraint parameters. For example, the local approaches have been used to predict changes in the apparent fracture toughness values across constraint levels caused by specimen geometries (such as $[14,15]$ ) and loading conditions (such as $[16,17]$ ). Gao and Dodds $[18,19]$ used the Weibull stress to scale macroscopic fracture toughness values across varying constraint levels in terms of $T$-stress. Qian et al. [20] comprised the difference in crack tip constraints by $T$ stress and $Q$ for cruciform specimen, compact tension specimen and three-point bending specimen with shallow and deep cracks and analyzed the probabilities of cleavage fracture based on Beremin model. It is necessary to investigate the general relationship between Weibull stress in local approaches and the fracture driving force and constraint parameters in macroscopic fracture mechanics.

The primary motivation of this work is to obtain semi-analytical expressions for the Weibull stress in terms of the macroscopic crack driving forces and the crack tip constraint parameters.

The layout of this paper is as follows. Section 2 describes the theoretical background, including the Weibull stress and constraint parameters. Section 3 describes the FE models used in determining the local parameters and Section 4 gives the results of these parameters. Section 5 provides verification and discussions of the results. The main conclusions of this work are summarized in Section 6.

\section{Theoretical background}

\subsection{Weibull stress in local approach}

In 1983, the Beremin group [1] proposed a local model for cleavage fracture based on micromechanical and probabilistic analysis in the FPZ at the crack tip. The theory is that the local stress concentration and plastic deformation in the FPZ ahead of the crack tip due to the external loads promote dislocations and slip motions in the micro carbide particles and impurities existing in the material and cause the formation of cleavage microcracks. Due to the local inhomogeneity of the organization in the actual material, a large number of micro-cracks of various sizes, shapes and orientations formed in the fracture process zone under continuous plastic deformation are randomly distributed. Therefore, the material's ability to resist against fracture and the distribution of shapes, sizes and orientations of the carbide particles or impurities in the material determine the failure probability. In its original form, the cumulative fracture probability, $P_{\mathrm{f}}$, is described by a two-parameter Weibull distribution as follows.

$P_{\mathrm{f}}=1-\exp \left[-\left(\frac{\sigma_{\mathrm{w}}}{\sigma_{\mathrm{u}}}\right)^{m}\right]$

where $m$ and $\sigma_{\mathrm{u}}$ are the Weibull modulus which depend on the statistical distributions of sizes, shapes and orientations of micro-cracks in the material and $\sigma_{\mathrm{w}}$ is the Weibull stress which can be defined as follows in Eq. (2).

$\sigma_{\mathrm{w}}^{m}=\frac{1}{V_{0}} \int_{V_{\mathrm{p}}}\left(\sigma_{1}\right)^{m} d V$.

where $V_{0}$ defines the reference volume and $V_{\mathrm{p}}$ is the FPZ and can be defined by the following equation.

$\sigma_{1} \geqslant \lambda \sigma_{0}$,

where $\sigma_{0}$ represents uniaxial, tensile yield stress and the coefficient $\lambda$ defines the size of FPZ. In general, $\lambda=2$ is adopted [21,22].

\subsection{Constraint parameters in macroscopic fracture mechanics}

\subsubsection{T-stress for elastic materials}

The near crack-tip stress fields in an isotropic elastic material can be expressed, following Williams [23], as an infinite power series like

$\sigma_{i j}(r, \theta)=A_{1} r^{1 / 2} f_{i j}^{1}(\theta)+A_{2} f_{i j}^{2}(\theta)+A_{3} r^{1 / 2} f_{i j}^{3}(\theta)+\ldots$.

where $r$ and $\theta$ are the polar coordinates with the origin at the crack tip, $f_{\mathrm{ij}}(\theta)$ are the location parameters at the crack tip as a function of $\theta$ and $A_{k} \cdot(k=1,2,3, \ldots)$ are constants depending on the load types. Classical fracture mechanics theory normally neglects all but the singular term, which results in a single-parameters description of the near-tip fields. Although the third and higher terms in the Williams solution, which have positive exponents on $r$, vanish at the crack tip, the second term remains finite. It turns out that this second term can have a profound effect on the FPZ shape and stresses deep inside the fracture process zone. For a crack in an isotropic elastic material subject to plane strain Model I loading, the first two terms of the Williams solution can be expressed as

$\sigma_{i j}=\frac{K_{I}}{\sqrt{2 \pi r}} f_{i j}(\theta)+\left[\begin{array}{ccc}T & 0 & 0 \\ 0 & 0 & 0 \\ 0 & 0 & v T\end{array}\right]$. 
where $T$ represents the $T$-stress, a uniform stress in the $x$ direction, $K_{\mathrm{I}}$ is Model I SIF and $v$ is Poisson's ratio of material. From Eq. (5), the SIF, $K$, and the $T$-stress characterize the elastic crack-tip stress fields. Both $K$ and $T$ are directly proportional to the load applied to a cracked geometry, the biaxiality ratio $\beta$ is defined by $[24,25]$

$\beta=\frac{T \sqrt{\pi a}}{K}$.

\subsection{2. $Q$ factor for elastic-plastic materials}

$T$-stress is an elastic parameter and $K$ - $T$ theory is valid for elastic materials. For elastic-plastic materials, O'Dowd and Shih et al. [12,13] proposed a new constraint parameter, $Q$ factor, and proposed a $J-Q$ theory to describe the crack tip stress field for a crack in an elasticplastic material body. The $\mathrm{Q}$ factor is defined by Eq. (7) below.

$Q=\frac{\sigma_{\mathrm{yy}}-\left(\sigma_{\mathrm{yy}}\right)_{\mathrm{SSY}}}{\sigma_{0}}$ at $\quad r=\frac{2 J}{\sigma_{0}}$ and $\theta=0$.

where $\sigma_{\mathrm{yy}}$ is the open crack stress of the crack tip, $\left(\sigma_{\mathrm{yy}}\right)_{\mathrm{SSY}}$ is the opening stress of the crack tip under the small-scale yield (SSY) and plane strain conditions and $J$ is the elastic-plastic fracture parameter.

\section{Finite element models}

To investigate the effect of constraint on Weibull stress, numerical analysis is performed using FE method. The modified boundary layer (MBL) model (see Fig. 1(a)) is used to generate the stress filed under various load conditions for deriving the solutions. Weibull stress is computed based on Eq. (2) by drawing the principle stress at the integration points of elements in the PFZ. In addition, compact tension (CT) specimen and single-edge cracked plate under three-point bending (SEB) specimens used in standard fracture toughness test are also analyzed. The ABAQUS [22] FE software is used in all FE analyses of this work. The 8-noded plain strain element with reduced integration,

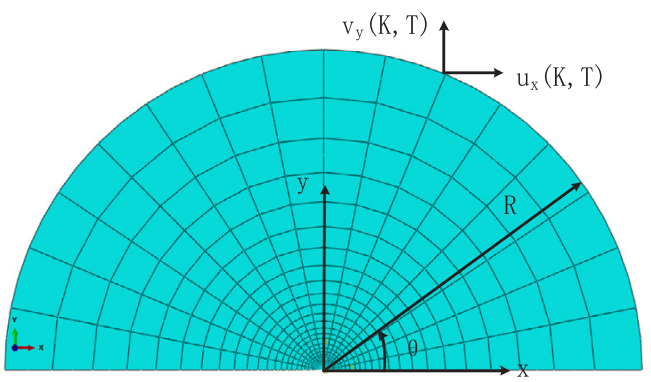

(a)

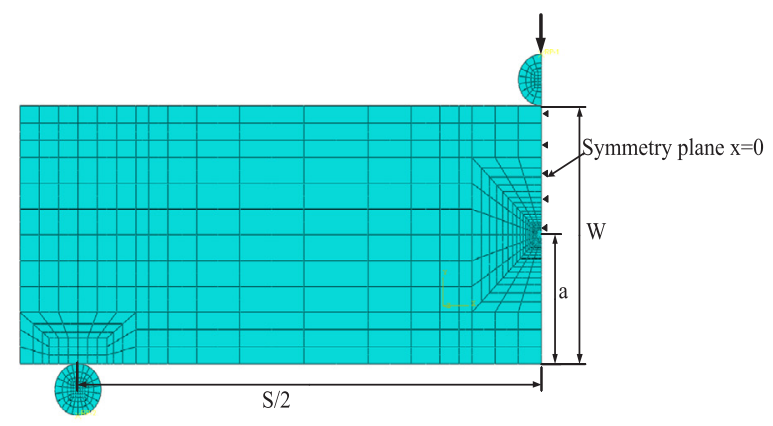

(c) the ABAQUS element type CPE8R, is used to simulate the three models. A sample of FE mesh for each model used in the analysis is shown in Fig. 1 .

For elastic-plastic analyses, the Ramberg-Osgood stress-strain law defined in Eq. (8) below is used.

$\frac{\varepsilon}{\varepsilon_{0}}=\frac{\sigma}{\sigma_{0}}+\alpha\left(\frac{\sigma}{\sigma_{0}}\right)^{\mathrm{n}}$.

where $\alpha$ is a material constant, $n$ is the hardening exponent, $\sigma_{0}$ is a normalizing stress which is close to the $2 \%$ plastic strain off-set stress and $\varepsilon_{0}=\sigma_{0} / E$ where $E$ is Young's modulus. The parameter studies here consider three sets of material properties with $n=5\left(E / \sigma_{0}=800\right)$, $n=10\left(E / \sigma_{0}=500\right), n=10\left(E / \sigma_{0}=300\right)$ and material coefficient $\alpha=1$. The linear elastic material properties are considered with $E=200 \mathrm{GPa}$ and $v=0.3$.

\subsection{Modified boundary layer model}

The leading two terms in the classical William's expression [23], shown in Eq. (5), demonstrate the similitude in the linear elastic crack front fields for crack fronts subjected to identical $K$ and $T$-stress values. Conventional MBL model was firstly proposed by Rice [27] that a tiny initial crack is contained in a large semicircle and a displacement field due to the given $K$ and $T$ values is applied to the edge of the boundary layer under certain conditions. This model can accurately reflect the displacement stress and strain field at the crack front.

The applied linear elastic displacement field for plane strain conditions is described by the following equation.

$u_{1}(r, \theta)=K_{I} \frac{1+v}{E} \sqrt{\frac{r}{2 \pi}} \cos \left(\frac{\theta}{2}\right)(3-4 v-\cos \theta)+T \frac{1-v^{2}}{E} r \cos \theta$
$u_{2}(r, \theta)=K_{I} \frac{1+v}{E} \sqrt{\frac{r}{2 \pi}} \sin \left(\frac{\theta}{2}\right)(3-4 v-\cos \theta)+T \frac{1+v^{2}}{E} r \sin \theta$

where $u_{1}$ and $u_{2}$ represent the displacement components along $\mathrm{x}$ -

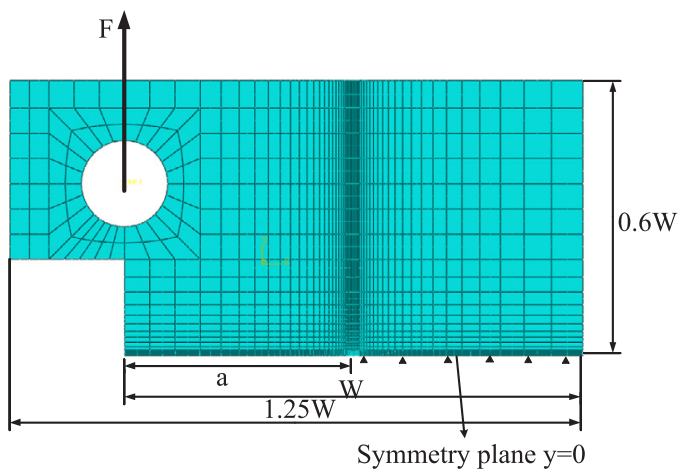

(b)

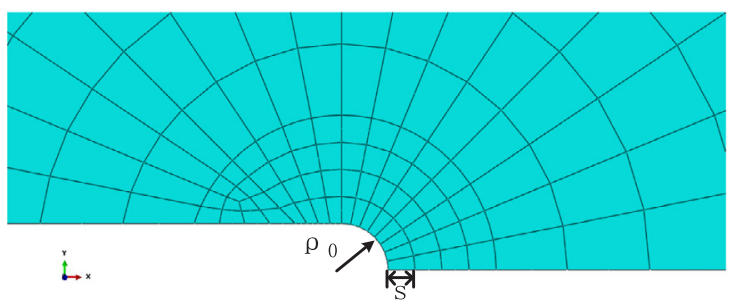

(d)

Fig. 1. Finite element models (a) MBL model (b) CT model (c) SEB model (d) enlarged view of the crack front. 
direction and y-direction, respectively. Fig. 1(d) shows an enlarged view of the crack tip simulated with a small notch radius, $\rho$. In order to verify the effect of the notch radius on the stress field, various notch radius values, $2 \mu \mathrm{m}, 3 \mu \mathrm{m}$ and $4 \mu \mathrm{m}$, are analyzed. The basic scale of MBL model used in the analyses is $r / \rho_{0}=10^{6}$.

\subsection{CT and SEB models}

In order to study the influence of the high constraint model on the crack tip stress field, CT specimens are analyzed. As shown in Fig. 1(b), a 2D FE model of half specimen with a width of $W=50 \mathrm{~mm}$ and height of $0.6 \mathrm{~W}$ is created considering the symmetry of geometry and load. The ratio $a / W$ between the crack length $a$ and the width $W$ is set to be 0.5 . The symmetrical boundary conditions are applied at the symmetry plane of the specimen. A concentrated force, $F$, is applied on a reference point located in the middle of the loading hole, which is coupled with the nodes along the up-half loading hole.

The SEB specimens are also modeled in order to study the crack tip stress field with low constraint levels and verify the proposed Weibull stress expressions considering constraint levels. As shown in Fig. 1(c), 2D plane strain models are analyzed with a loading span $S=200 \mathrm{~mm}$, the specimen height $W=50 \mathrm{~mm}$ and various crack length, $a$.

\subsection{Computation of Weibull stress using the FE method}

Consider a specimen thickness $B$, the Weibull stress can be calculated, based on Eq. (2), using the following equation.

$\sigma_{\mathrm{w}}^{m}=\sum_{n_{\mathrm{e}}} \frac{B}{V_{0}} \int_{-1}^{1} \int_{-1}^{1} \sigma_{1}^{m}|J| d \xi d \eta$

where $n_{\mathrm{e}}$ is the number of elements inside the FPZ and $|J|$ denotes the determinant of the standard coordinate Jacobin between deformed Cartesian $(x, y)$ and element local coordinate system $(\xi, \eta)$. Because the size of the reference volume $V_{0}$ does not affect the final Weibull stress failure probability in theory, the reference volume $V_{0}$ here is taken as $1 \mathrm{~mm}^{3}$ for the convenience of calculation.

\section{Weibull stress with constraint parameters}

\subsection{Weibull stress solution with $T$-stress}

The stress fields near a notch in an elastic material for loads resulted in a $K$ - $T$ field is given by

$$
\left(\begin{array}{c}
\sigma_{x} \\
\sigma_{y} \\
\tau_{x y}
\end{array}\right)=\frac{K}{\sqrt{2 \pi r}} \frac{\rho}{2 r}\left(\begin{array}{c}
-\cos \frac{3 \theta}{2} \\
\cos \frac{3 \theta}{2} \\
-\sin \frac{3 \theta}{2}
\end{array}\right)+\frac{K}{\sqrt{2 \pi r}} \cos \frac{\theta}{2}\left(\begin{array}{c}
1-\sin \frac{\theta}{2} \sin \frac{3 \theta}{2} \\
1+\sin \frac{\theta}{2} \sin \frac{3 \theta}{2} \\
\sin \frac{\theta}{2} \cos \frac{3 \theta}{2}
\end{array}\right)+\left(\begin{array}{l}
T \\
0 \\
0
\end{array}\right),
$$

where $\rho$ is the radius of the notch (see Fig. 2). The maximum principal stress for the elastic stress fields given in Eq. (11) can be expressed as

$\sigma_{1}$

$$
\begin{aligned}
= & \frac{K}{\sqrt{2 \pi r}}\left(\cos \frac{\theta}{2}+\frac{1}{2} \beta^{\prime}\right. \\
& \left.+\frac{1}{2} \sqrt{\left(\frac{\rho}{r}\right)^{2}+\sin ^{2} \theta-2 \beta^{\prime}\left(\frac{\rho}{r} \cos \frac{3 \theta}{2}+\sin \theta \sin \frac{3 \theta}{2}\right)+\beta^{\prime 2}}\right),
\end{aligned}
$$

where $\beta^{\prime}=\frac{T \sqrt{2 \pi r}}{K}$. Then the Weibull stress can be obtained by substituting Eq. (12) into Eq. (2) and may be simplified to

$\sigma_{\mathrm{w}}^{m}(K, T)=\frac{2}{L^{2}} \int_{0}^{\pi} \int_{R_{1}(\theta)}^{R_{2}(K, T, \theta)} \sigma_{1}^{m}(r, \theta) r d r d \theta$,

where the length parameter $L$ is defined as $L=\sqrt{V_{0} / B}$. The integral range is from $R_{1}$ to $R_{2}$, where $R_{1}$ is the notch surface (see Fig. 2) and can be expressed, based on the coordinate system shown in Fig. 2, as

$R_{1}(\theta)=\left\{\begin{array}{cl}\frac{\rho}{2}\left(-\cos \theta+\sqrt{\cos ^{2} \theta+3}\right) \quad 0 \leqslant \theta \leqslant(\pi-\arctan 2) \\ \frac{\rho}{\sin (\pi-\theta)} \quad(\pi-\arctan 2) \leqslant \theta<\pi\end{array}\right.$.

The upper limit of integration, $R_{2}$, is on the FPZ boundary, which can be determined by substituting Eq. (12) into Eq. (3) and solving for $r$. Then the integral in Eq. (13) can be evaluated when $R_{1}$ and $R_{2}$ are available. However, a closed form solution could not be obtained and numerical integral has to been used instead.

To compare with the results from Lei et al. [8], a solution with $T=0$ is considered for Weibull modulus parameters $m=10,15,20$, and 25, as shown in Fig. 3. It can be found that the results are agreed with well.

\subsection{Weibull stress with $Q$ parameter}

For a notch with elastic-plastic material, there is not a suitable solution for stress field. Therefore, a finite element analysis with MBL model was carried out to assist in the formulation of a suitable expression. In the MBL model, the displacement field calculated by Eq. (9) is applied to obtain $J$-integral and $Q$ value.

\subsubsection{Formulation inference for Weibull stress with $Q$ parameter}

The effect of $Q$ on Weibull stress is studied with MBL models with elastic-plastic material. A Weibull stress ratio for different constraints is defined as:

$\sigma_{\mathrm{w}}=\Phi(J, Q)\left(\sigma_{\mathrm{w}}\right)_{T=0}$.

where $\left(\sigma_{\mathrm{W}}\right)_{T=0}$ means the Weibull stress under $T=0$. The scale factor $\Phi(J, Q)$ describes the deviation of Weibull stress under different constraints conditions. It is related to material properties $\left(n, E / \sigma_{0}, \alpha, v, m\right)$ and crack tip stress field $(J-Q)$.

For elastic plastic material, it should be noted that the effect of notch radius can be eliminated, as shown in Fig. 4. Weibull stress varied with $J$-integrity is calculated using MBL model with different notch radius $\rho=2 \mu \mathrm{m}, 3 \mu \mathrm{m}$ and $4 \mu \mathrm{m}$. There is no significant difference in the stress field near the crack tip which is surrounded by a local plastic zone. This initial root radius could facilitate the numerical convergence at large plastic deformation near the crack tip. Therefore, the subsequent derivations use a notch radius of $3 \mu \mathrm{m}$, which is coincident with several literatures $[13,17]$.

The effect of constraint parameter $Q$ on Weibull stress is preliminary investigated with different stress fields, as shown in Fig. 5. It can be clearly found that the scale factor increases linearly with the constraint parameter $Q$ for the material with Weibull module $m=30$ and hardening exponent $n=10$. In addition, the scale factor exhibits different slopes with increasing $Q$ at different load levels (different $J$ values).

The loading effect on Weibull stress is analyzed in form of $J$-integral. Fig. 6 shows Weibull stress with $J$-integral under different constraints. A

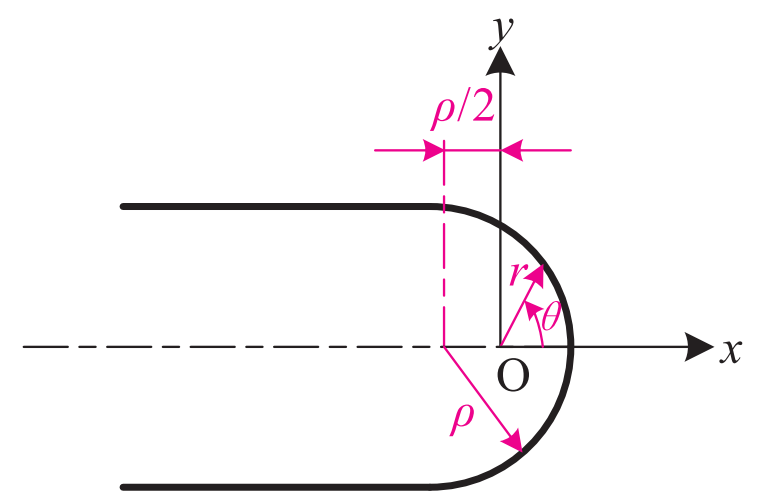

Fig. 2. Coordinate system used for notch crack. 


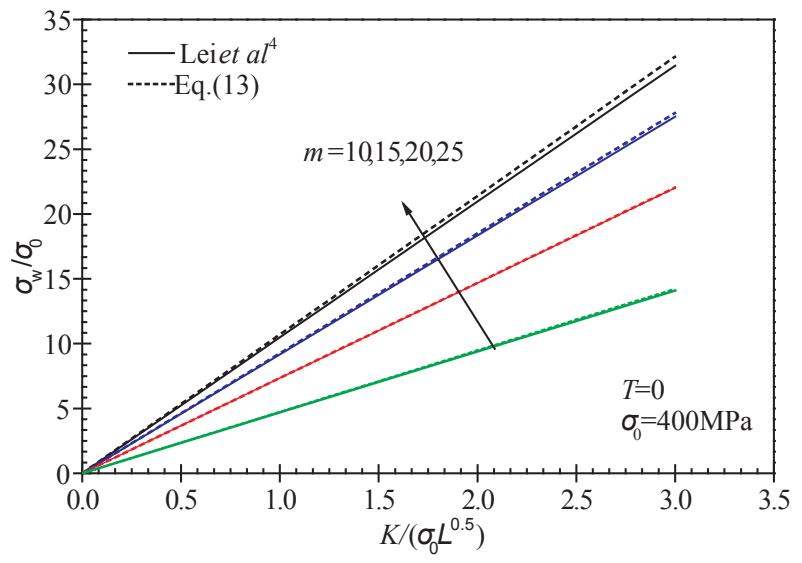

Fig. 3. Comparison with Lei's solution for notch under $T=0$.

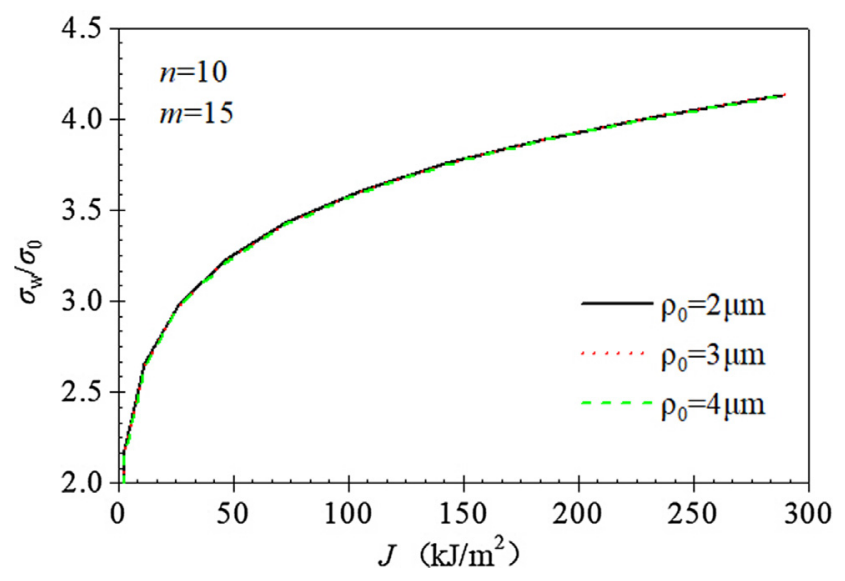

Fig. 4. Effect of notch radius on Weibull stress for elastic plastic material.

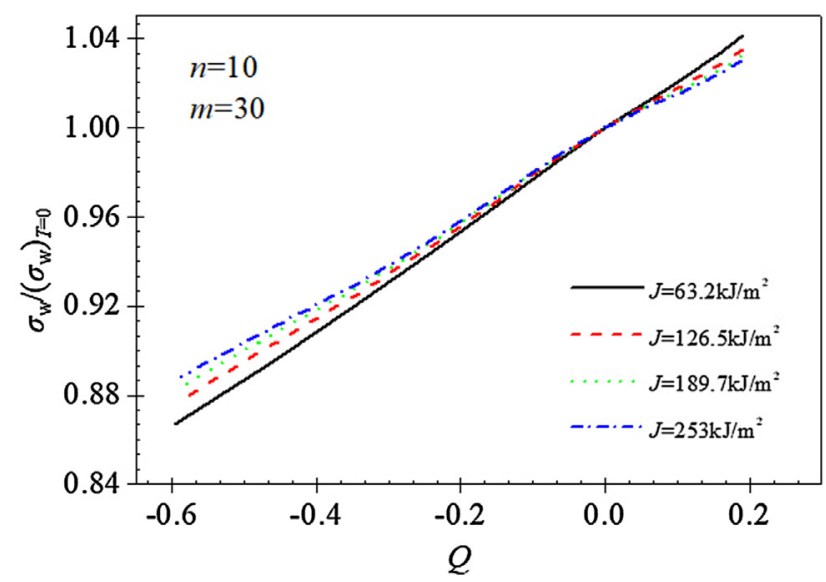

Fig. 5. Effect of $Q$-stress on for elastic plastic material.

similar trend that Weibull stress increases with $J$-integral can be found for the two constraint conditions. A form of exponent may be used to express the trend. At the same time, the Weibull modulus $m$ has a great effect on Weibull.

According to the preliminary analysis, a formulation for Weibull stress with $Q$-stress is inferred as

$\Phi(J, Q)=1+\xi Q\left(\frac{\mathrm{J}}{\alpha \varepsilon_{0} \sigma_{0} L}\right)^{\frac{\zeta}{\mathrm{n}+1}}$.

where $\xi$ and $\zeta$ are coefficients which are functions of Weibull

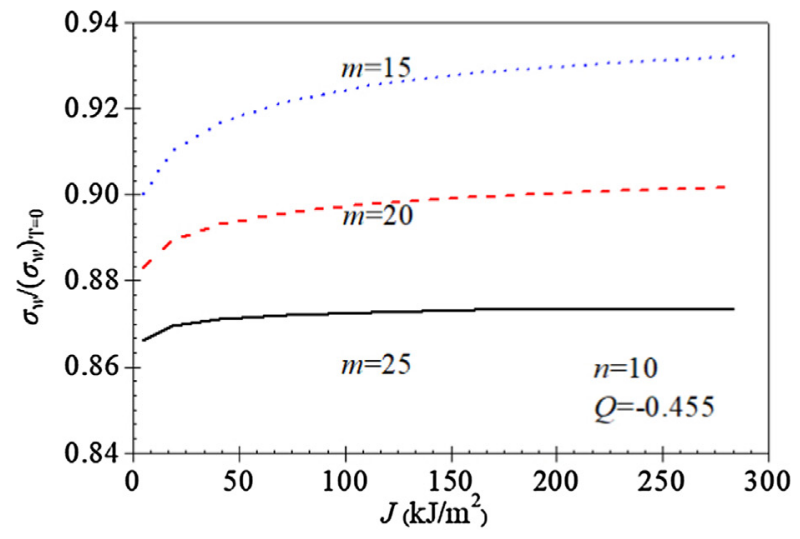

(a) $Q=-0.455$

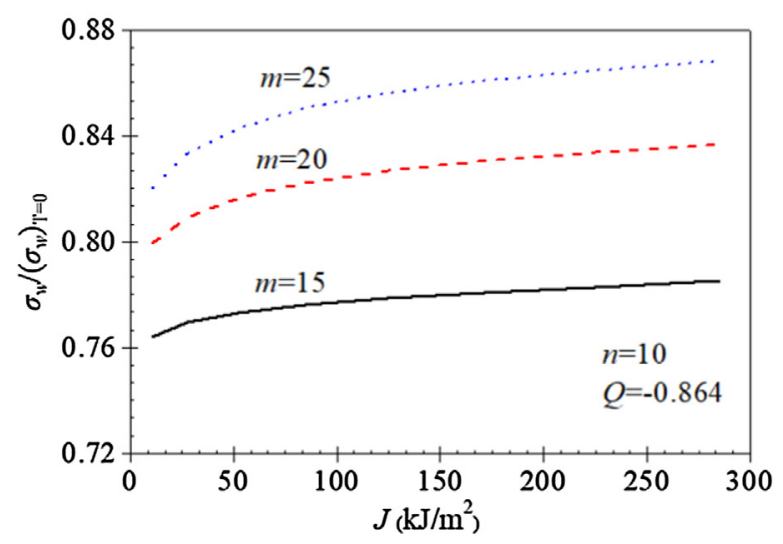

(b) $Q=-0.864$

Fig. 6. Weibull stress varied as $J$-integrity with different values of $Q$.

module $m$ and constraint parameter $Q$. In next section, we will discuss and give solutions of the two coefficients.

\subsubsection{Solutions of the coefficients}

Reexamining Eq. (17), the coefficient $\zeta /(\mathrm{n}+1)$ is the slope if taking logarithm of two sides of Eq. (17). Fig. 7 shows typical finite element results for $n=10$. It can be seen that the lines for different $Q$ values are parallel with the given Weibull modulus $m$. Therefore, it can be assumed that the coefficient $\zeta$ is independent with $Q$ value. However, the coefficient $\zeta$ will depend on the sign of $Q$, which is approved as shown in Fig. 5. There is a turning point for the function $\Phi$ with $Q$ value. For a high constraint level with positive $Q$ value, the value of $\Phi$ is larger than 1 . For negative $Q$ value, the value of $\Phi$ is smaller than 1 . Therefore, the coefficient $\zeta$ will be determined for positive and negative $Q$ values individually. Table 1 shows the value of coefficient $\zeta$ for different $m$ and $n$.

According to the coefficient $\zeta$ determined in Table 1 , the coefficient $\xi$ under different $Q$ values is further obtained, which is a function of $Q$, $m$ and $n$. Table 2 lists the value of coefficient $\xi$ for typical material values of $m$ and $n$. It is more convenient to calculate Weibull stress under $J$ and $Q$ from Table 2.

\section{Verifications and discussions}

In this section, the proposed Weibull stress solutions of Eq. (12) for elastic material and Eq. (12) for elastic-plastic material will be verified by finite element models including MBL model, CT model and SEB model. Then, we perform some discussions on the effect of constraint parameters on Weibull stress for linear-elastic materials and elasticplastic materials. 


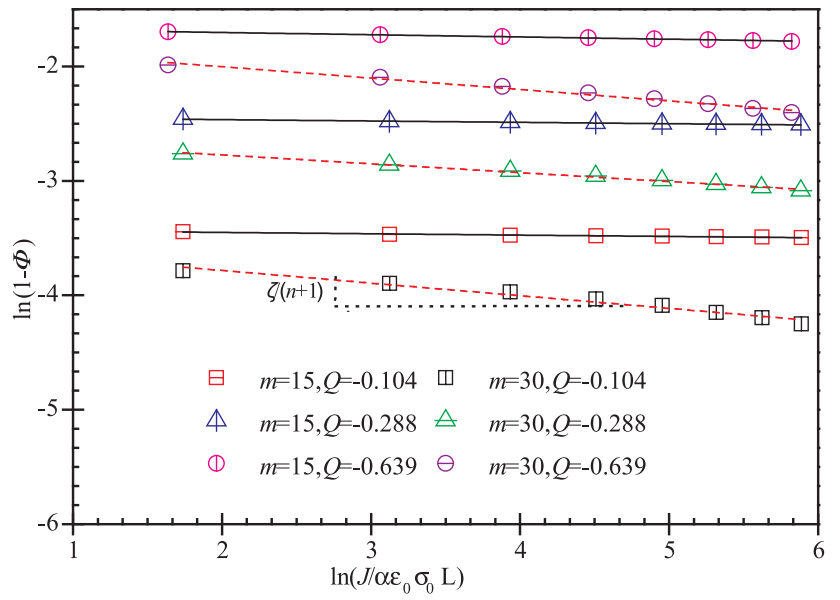

(a)

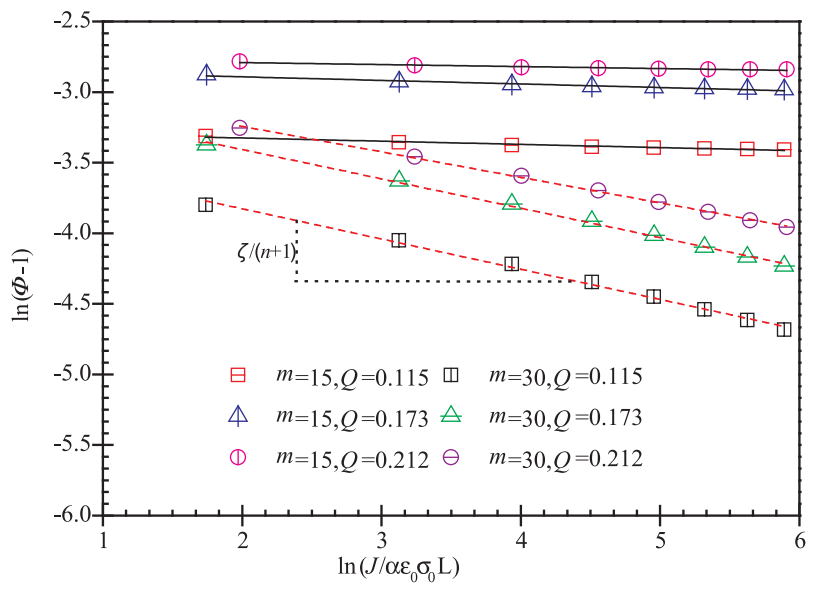

(b)

Fig. 7. A diagrammatic view of determining the coefficients from finite element analysis for $n=10$ (a) $Q<0$, (b) $Q>0$.

Table 1

Values of coefficient $\zeta$.

\begin{tabular}{llll}
\hline Material & $m$ & $Q<0$ & $Q>0$ \\
\hline$n=5$ & 15 & -1.0187 & -1.4220 \\
& 20 & -1.3114 & -1.8277 \\
& 25 & -1.4868 & -2.0487 \\
& 30 & -1.5755 & -2.1371 \\
& 40 & -1.6151 & -2.1247 \\
$n=10$ & 50 & -1.6746 & -2.2083 \\
& 15 & -0.1917 & -0.1411 \\
& 20 & -0.5460 & -0.8761 \\
& 25 & -0.8366 & -1.5174 \\
& 30 & -1.0679 & -2.0506 \\
$n=20$ & 40 & -1.3714 & -2.7346 \\
& 50 & -1.4928 & -2.8129 \\
& 15 & -0.0222 & -0.0446 \\
& 20 & -0.0692 & -0.2807 \\
& 25 & -0.1697 & -0.6455 \\
& 30 & -0.3064 & -1.0995 \\
& 40 & -0.6191 & -2.1167 \\
& 50 & -0.8682 & -3.0158 \\
\hline
\end{tabular}

\subsection{Weibull stress for elastic material with T-stress}

\subsubsection{Verifications}

The finite element results obtained by MBL models are used to verify theoretical analytical solution for different notch radii $\rho$, as shown in Fig. 8. The solid lines present the proposed solution and the
Table 2

Values of coefficient $\xi$.

\begin{tabular}{|c|c|c|c|c|c|c|c|}
\hline Material & $Q$ & $m=15$ & $m=20$ & $m=25$ & $m=30$ & $m=40$ & $m=50$ \\
\hline \multirow[t]{9}{*}{$n=5$} & -1 & 0.2454 & 0.2589 & 0.2586 & 0.2491 & 0.2210 & 0.2119 \\
\hline & -0.8 & 0.2205 & 0.2042 & 0.1904 & 0.1788 & 0.1609 & 0.1483 \\
\hline & -0.6 & 0.2213 & 0.1974 & 0.1802 & 0.1682 & 0.1536 & 0.1409 \\
\hline & -0.4 & 0.2356 & 0.2146 & 0.1984 & 0.1862 & 0.1699 & 0.1586 \\
\hline & -0.2 & 0.2509 & 0.2323 & 0.2157 & 0.2015 & 0.1803 & 0.1700 \\
\hline & 0.05 & 0.2530 & 0.2188 & 0.1912 & 0.1693 & 0.1406 & 0.1276 \\
\hline & 0.1 & 0.2492 & 0.2077 & 0.1755 & 0.1512 & 0.1210 & 0.1067 \\
\hline & 0.15 & 0.2436 & 0.1929 & 0.1551 & 0.1280 & 0.0965 & 0.0805 \\
\hline & 0.2 & 0.2359 & 0.1741 & 0.1297 & 0.0992 & 0.0666 & 0.0485 \\
\hline \multirow[t]{9}{*}{$n=10$} & -1 & 0.2835 & 0.2634 & 0.2494 & 0.2401 & 0.2297 & 0.2206 \\
\hline & -0.8 & 0.2935 & 0.2758 & 0.2638 & 0.2560 & 0.2468 & 0.2366 \\
\hline & -0.6 & 0.2967 & 0.27818 & 0.2655 & 0.2572 & 0.2474 & 0.2368 \\
\hline & -0.4 & 0.2984 & 0.27724 & 0.2632 & 0.2544 & 0.2453 & 0.2350 \\
\hline & -0.2 & 0.3041 & 0.2799 & 0.2655 & 0.2583 & 0.2542 & 0.2452 \\
\hline & 0.05 & 0.2225 & 0.2258 & 0.2314 & 0.2372 & 0.2407 & 0.2189 \\
\hline & 0.1 & 0.3099 & 0.2919 & 0.2825 & 0.2782 & 0.2719 & 0.2462 \\
\hline & 0.15 & 0.3393 & 0.3136 & 0.2980 & 0.2889 & 0.2770 & 0.2503 \\
\hline & 0.2 & 0.3107 & 0.2908 & 0.2778 & 0.2693 & 0.2559 & 0.2312 \\
\hline \multirow[t]{8}{*}{$n=20$} & -1 & 0.3583 & 0.3229 & 0.3008 & 0.2889 & 0.2817 & 0.2745 \\
\hline & -0.8 & 0.3581 & 0.3258 & 0.3056 & 0.2945 & 0.2874 & 0.2809 \\
\hline & -0.6 & 0.3559 & 0.3264 & 0.3073 & 0.2964 & 0.2881 & 0.2814 \\
\hline & -0.4 & 0.3555 & 0.3270 & 0.3078 & 0.2958 & 0.2847 & 0.2767 \\
\hline & -0.2 & 0.3603 & 0.3300 & 0.3085 & 0.2939 & 0.2781 & 0.2673 \\
\hline & 0.05 & 0.3606 & 0.3188 & 0.2912 & 0.2744 & 0.2595 & 0.2465 \\
\hline & 0.1 & 0.3692 & 0.3242 & 0.2943 & 0.2758 & 0.2584 & 0.2423 \\
\hline & 0.15 & 0.3991 & 0.3462 & 0.3106 & 0.2881 & 0.2644 & 0.2403 \\
\hline
\end{tabular}

scatter points present the finite element solution. The $T$-stress of $0.5 \sigma_{0}$ is applied and Weibull module $m=15$ is assumed in this case. Weibull stress under a series of remote loading in form of $K$ is obtained. It can be clearly seen that the proposed analytical solutions agree very well with finite element results. In addition, Weibull stress depends on the notch radius who can result in varied stress field. For different notch radii, comparison between the predicted results and the FE solutions shows that good agreement is obtained throughout.

Furthermore, two cases by common specimens (CT and SEB) used to evaluate fracture toughness of material are also carried out. A typical crack size is selected to investigate the influence of constraint using $T$ stress, $a / W=0.5$ for CT model and $a / W=0.1$ for SEB model. The $T$ stress varied as remote loading is calculated first and shown in Fig. 9 for both models. As shown in Fig. 9, the T-stress shows a linear correlation of the remote loading with different gradients for the two models. The gradient can be described by the biaxiality ratio $\beta$ as defined in Eq. (6). It is calculated that $\beta=0.575$ for CT model with $a / W=0.5$ and $\beta=-0.777$ for SEB model with $a / W=0.1$. The reduce of $T$-stress for the shallow crack in SEB model is usually explained by the loss of constraint.

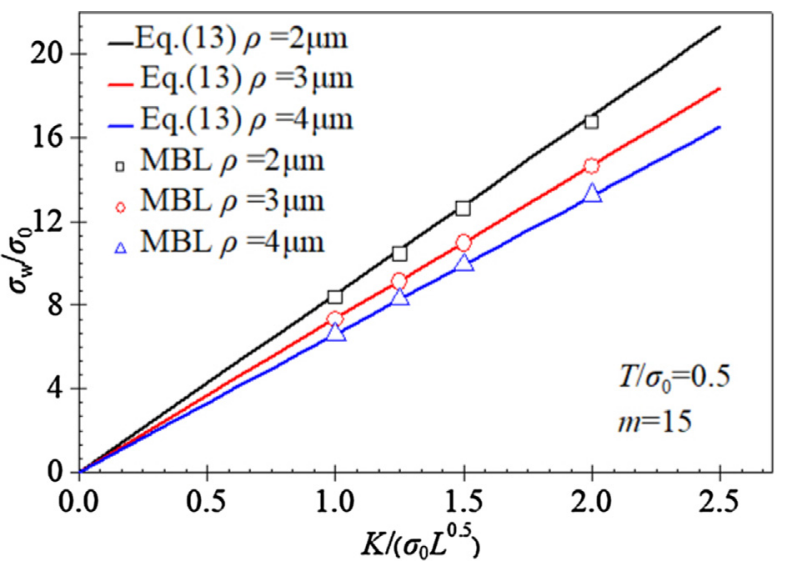

Fig. 8. Verification of Weibull stress solution for elastic material with $T$-stress using MBL models. 


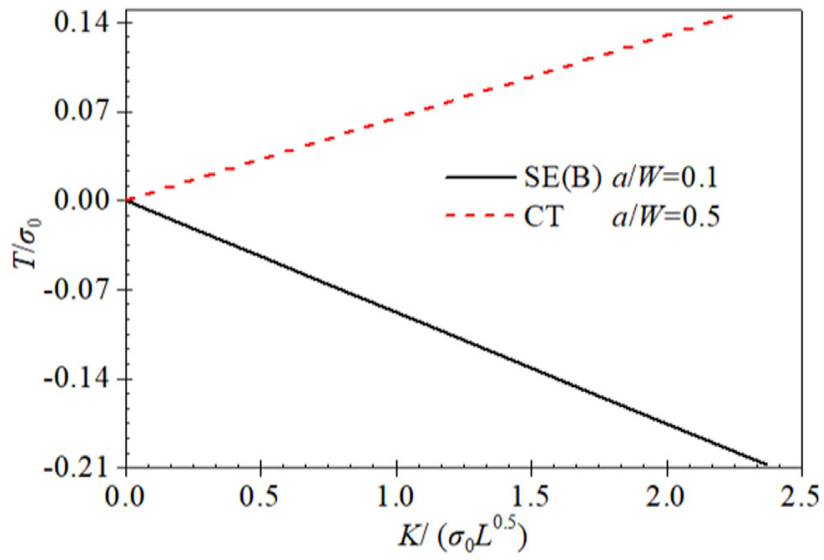

Fig. 9. T-stress for CT model and SEB model.

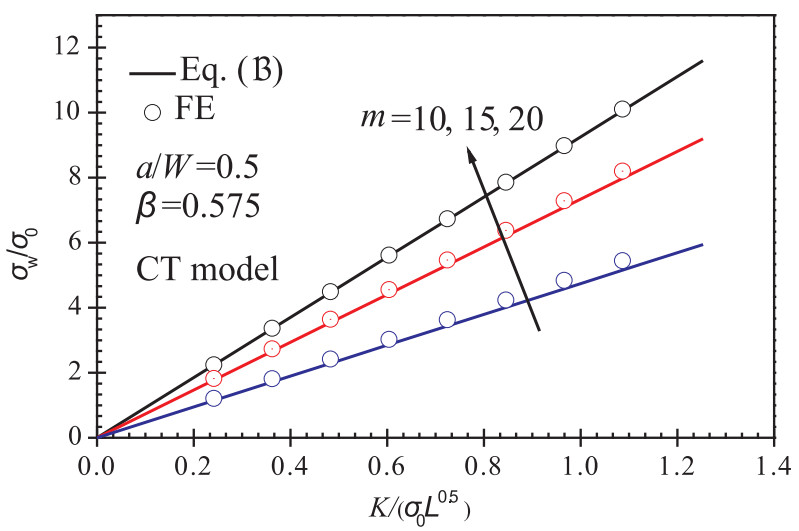

(a) CT model with $a / W=0.5$

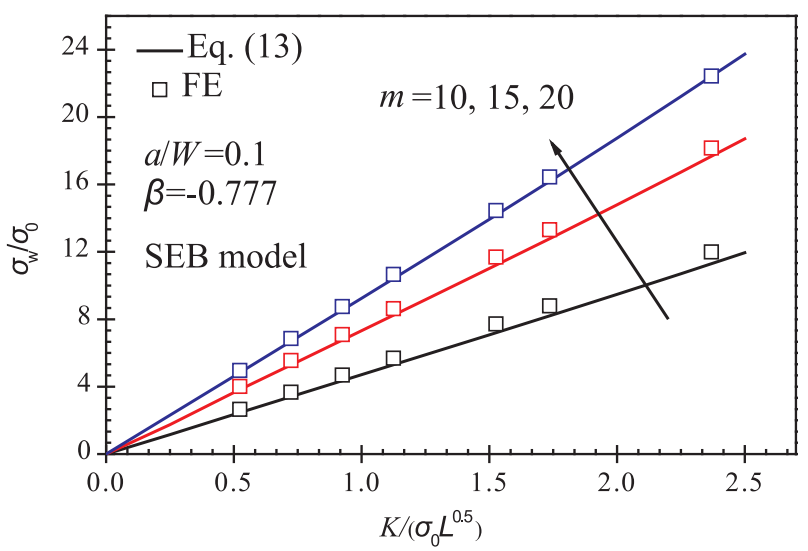

(b) SEB model with $a / W=0.1$

Fig. 10. Weibull stress estimated by the proposed solution and the finite element solution for CT and SEB models.

The Weibull stress is obtained with $\sigma_{0}=400 \mathrm{MPa}$ and $\lambda=2.0$ for the two cases, as shown in Fig. 10. The results for Weibull modulus $m$ of 10,15 , and 20 are also plotted in the figure. It can be seen that the proposed solution of Weibull stress agrees well with the finite element solution, particularly for a larger Weibull modulus $m$. A maximum relative error between the proposed and finite element solution is only $2 \%$ even for $m=10$. Therefore, the proposed solution can be used to estimate the Weibull stress with $T$-stress.

\subsubsection{Effect of T-stress}

Several cases are considered to investigate the influence of $T$-stress

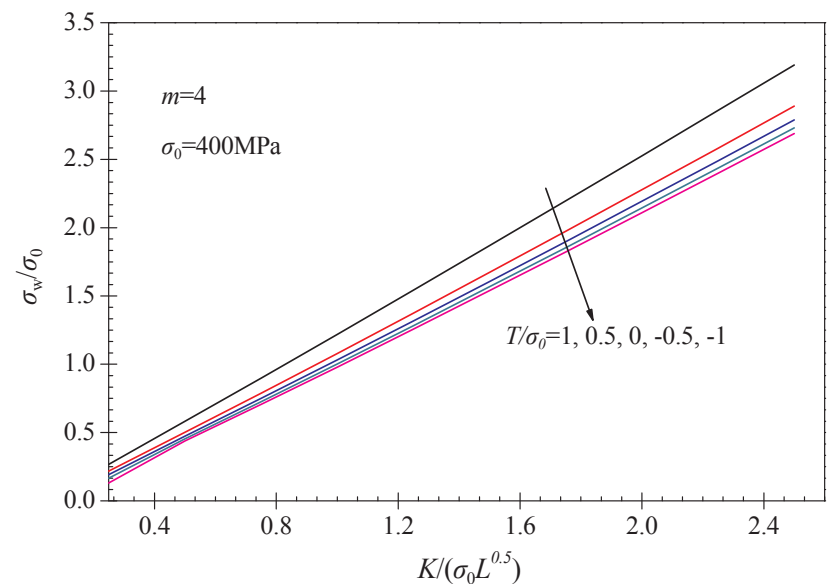

(a) $m=4$

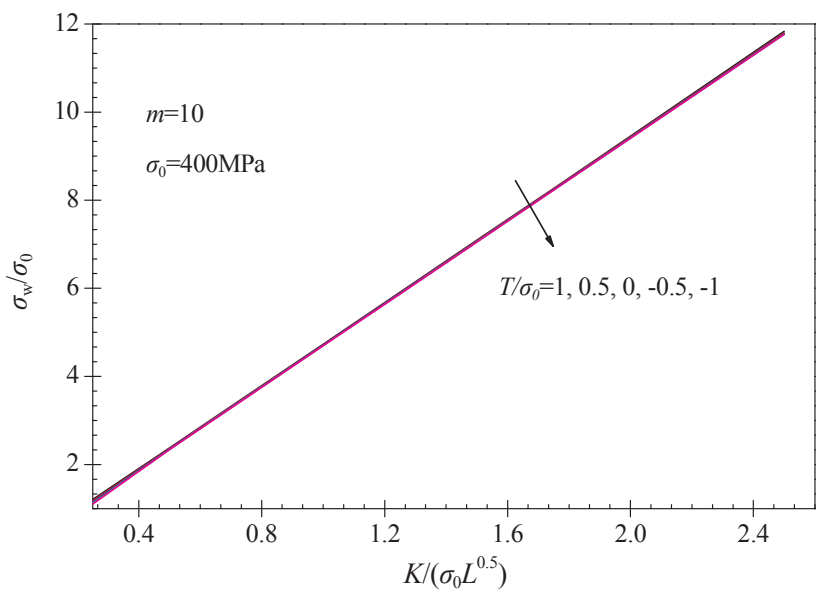

(b) $m=10$

Fig. 11. Weibull stress with $T$-stress estimated by the proposed solution for elastic material.

on Weibull stress, with $T / \sigma_{0}$ from -1.0 to 1.0 . The Weibull stress is computed by the proposed solution with Weibull modulus $m=4$ and 10 for elastic material, as shown in Fig. 11. It can be seen that $T$-stress has limited influence on the Weibull stress for a small Weibull modulus $m$, but the influence vanishes with the increased Weibull modulus. In other words, the Weibull stress with a large modulus is same for all $T$ stress. In this case, the constraint effect could not be interpreted by the proposed Weibull stress solutions. The mismatch may be attributed to the elastic material assumption in the derivation of Weibull stress solution. The stress near the notch remains in very high state without the plastic correction, which holds high weight in the integral interval as Eq. (13). The loss of constraint due to increasing plastic deformations cannot be captured well by the proposed solution on elastic material as reported in past research [11]. In the following section, a different result is obtained for elastic plastic material.

\subsection{Weibull stress for elastic plastic material with $Q$ parameter}

\subsubsection{Verifications}

First of all, the solution is rechecked using MBL model with other material parameters distinguished from those used in its construction. 3 cases with MBL model are applied to verify the solution with hardening exponent $n$ of 5, 10 and 20. The results are shown in Fig. 12. The constraint parameter $Q$ is calculated to be $-0.517,-0.639$ and -0.800 , respectively. It can be clearly seen that the proposed analytical solution agrees very well with these finite element solutions.

Then the specimen models are analyzed by elastic plastic finite 


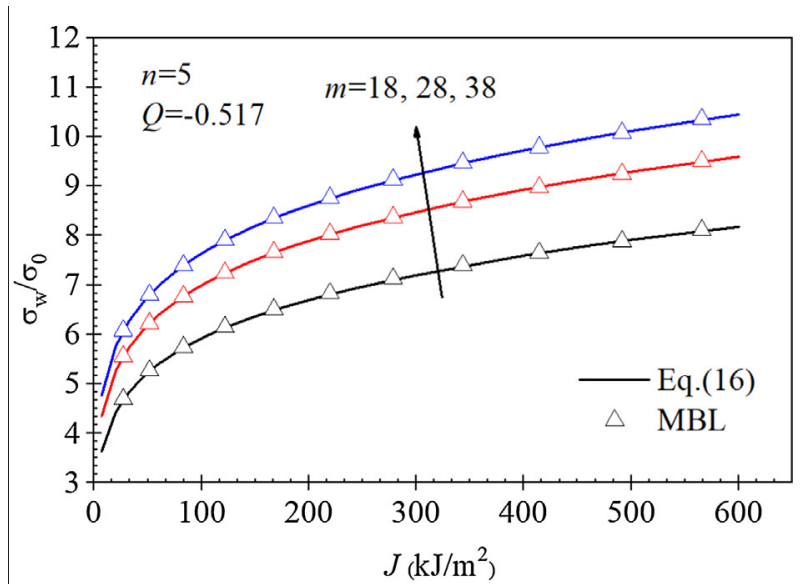

(a) $n=5$

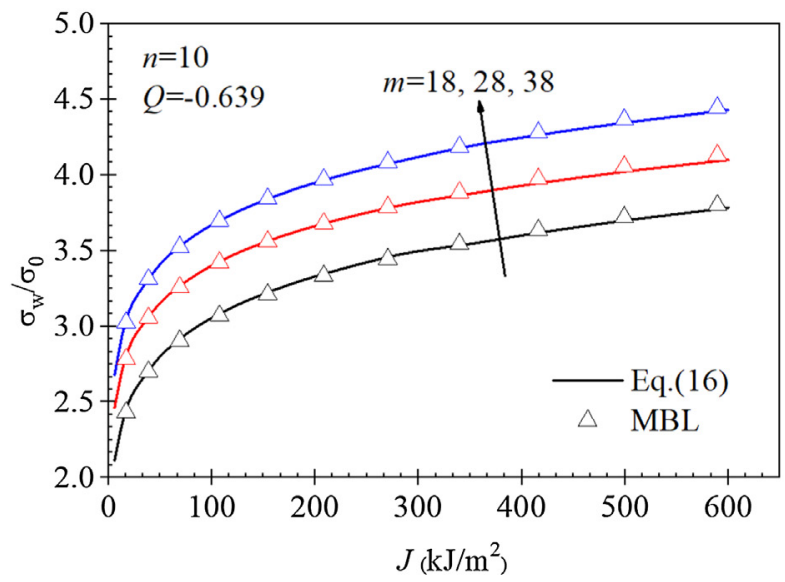

(b) $n=10$

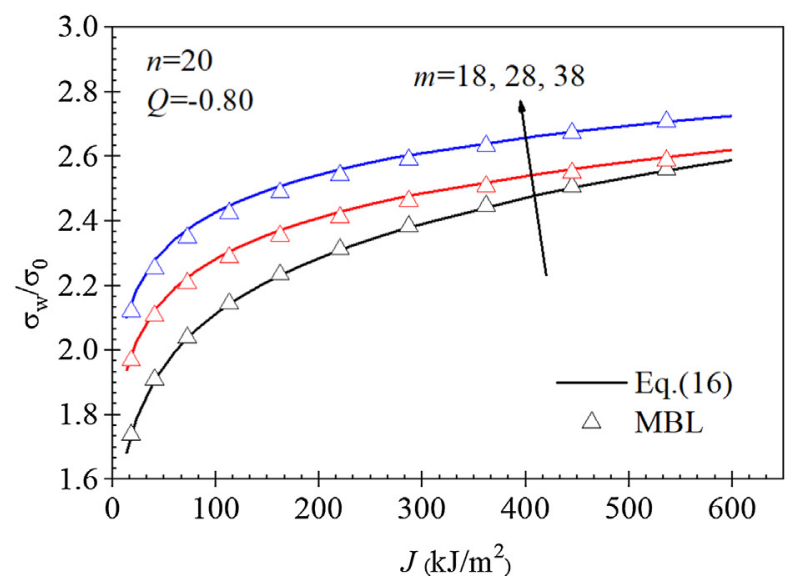

(c) $n=20$

Fig. 12. Verification of Weibull stress solution using MBL models for elastic plastic material with different hardening exponents.

element method. The constraint parameter is calculated first, as shown in Fig. 13. $J-Q$ trajectories for these specimens are plotted showing the effect of $a / W$ ratio and material hardening which similar trends have been reported in references [28,29]. Comparing Fig. 13(a) and (b), it can be found that shallow crack has greater constraint loss for a specified $J$-value. Beyond this, the constraint of CT specimen is higher than SEB specimen. Material hardening variations from $n=5$ to 10 have a small effect on $J-Q$ trajectories, but there is significant difference for

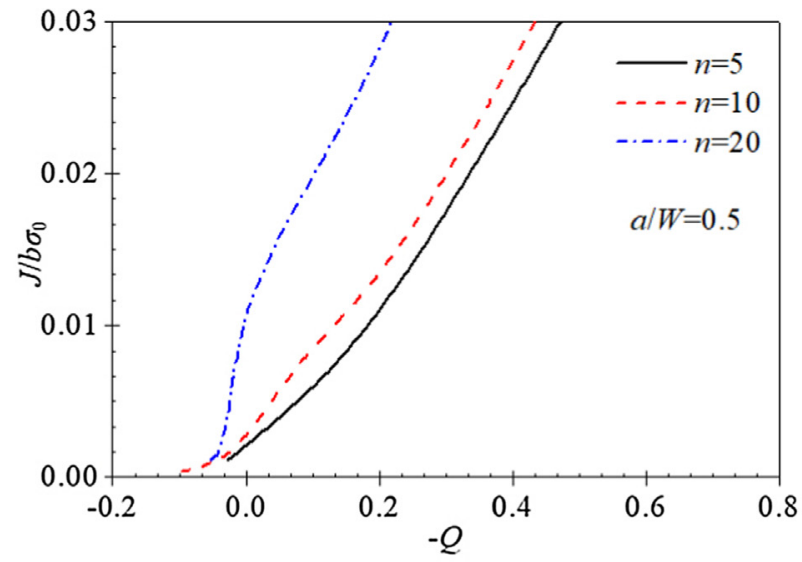

(a) SEB model with $a / W=0.5$

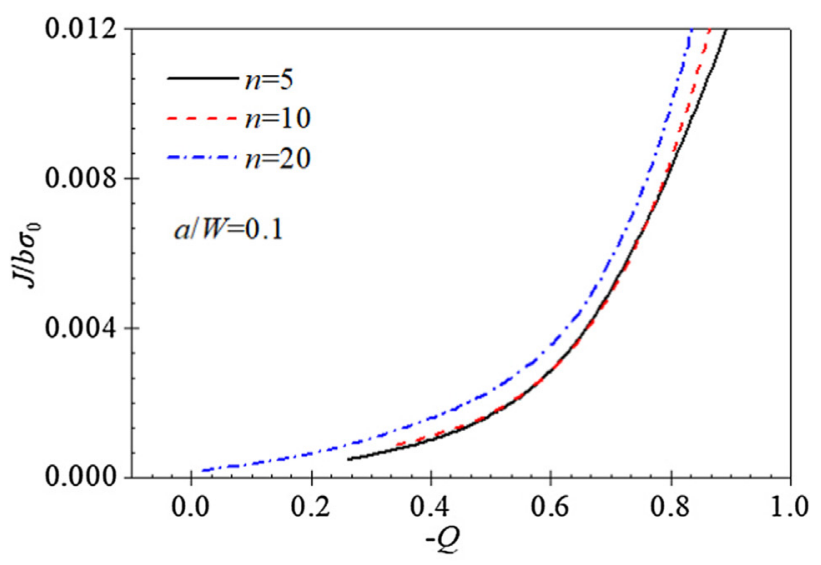

(b) SEB model with $a / W=0.1$

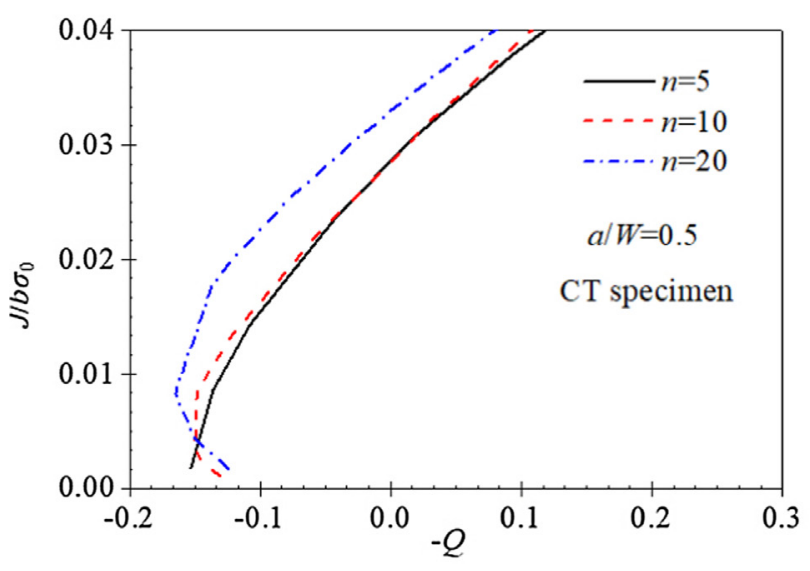

(c) CT model with $\mathrm{a} / \mathrm{W}=0.5$

Fig. 13. $J-Q$ trajectories for specimen models.

increased hardening $n=20$. Detailed discussions about constraint effects can be found in the work of Nevalainen and Dodds [30]. The $J$-Q trajectories will be used to determine $Q$-values in this work.

According to the $J$ - $Q$ trajectories, we can estimate Weibull stress by the proposed solution Eq. (22) for specified $J$-values. Fig. 14 provides comparisons of Weibull stress by the proposed solution and finite element results for specimen models. It can be clearly seen that the proposed semi-analytical solution agrees very well with finite element solution. 


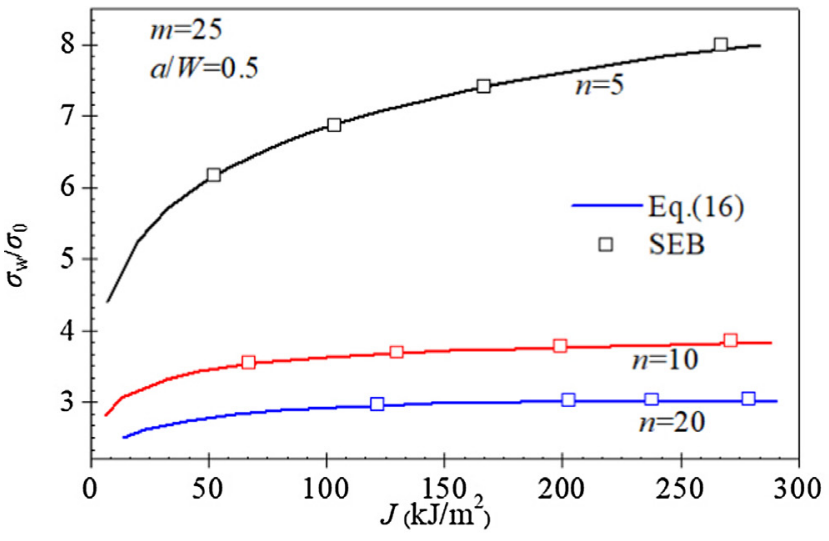

(a) SEB with $a / W=0.5$ and $m=25$

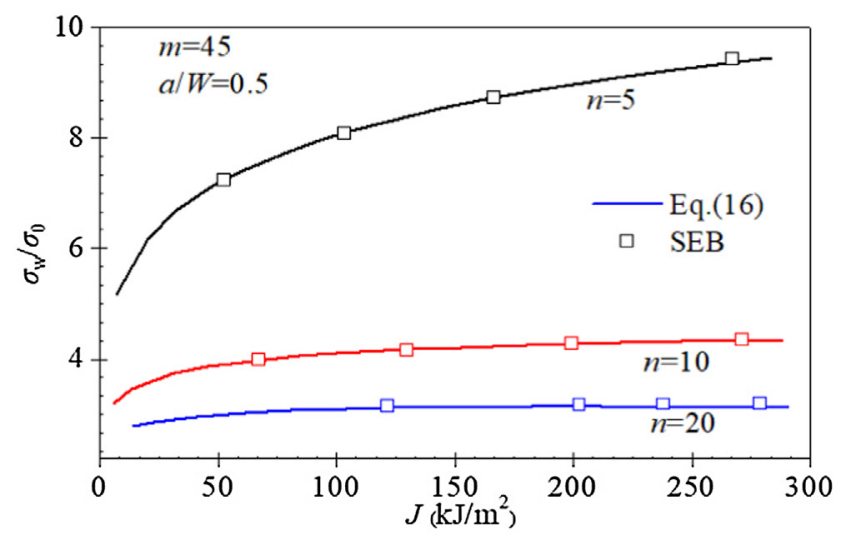

(b) SEB with $a / W=0.5$ and $m=45$

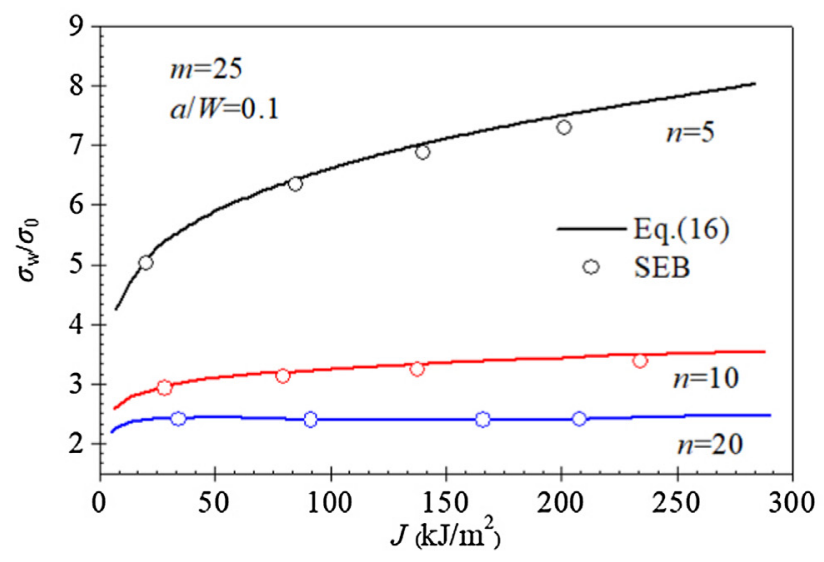

(c) SEB with $a / W=0.1$ and $m=25$

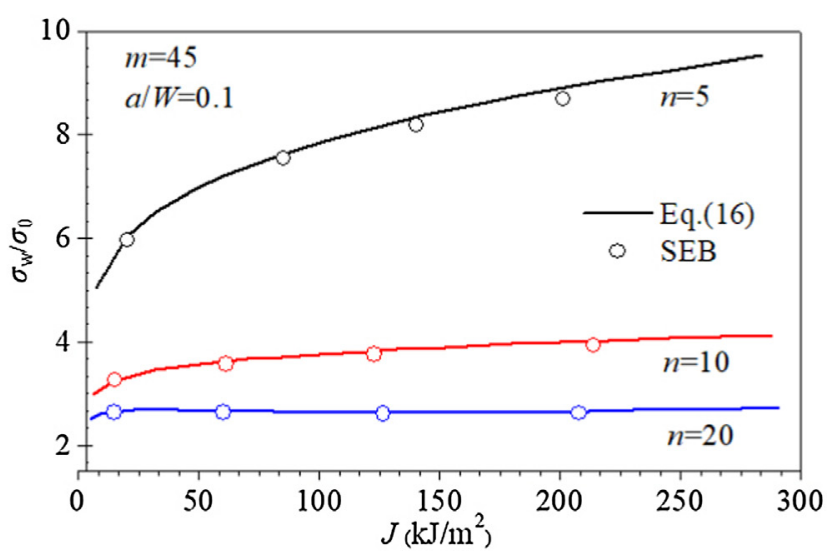

(d) SEB with $a / W=0.1$ and $m=45$

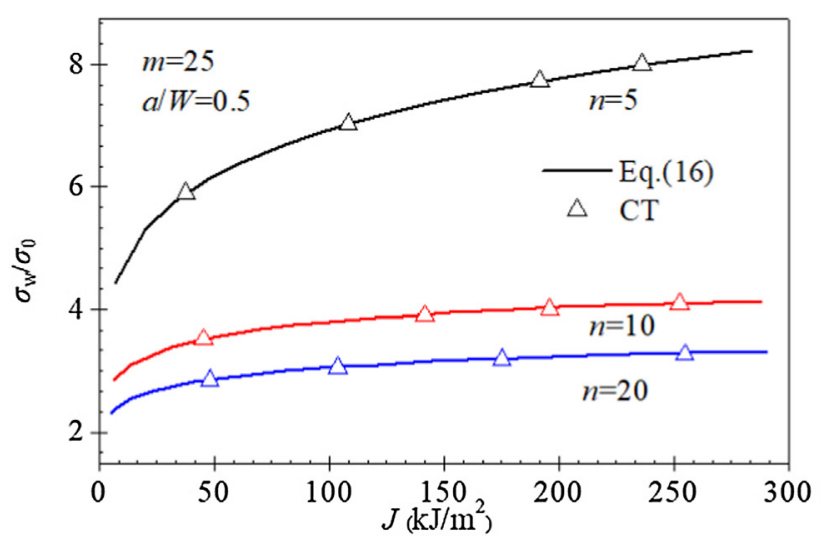

(e) CT with $a / W=0.5$ and $m=25$

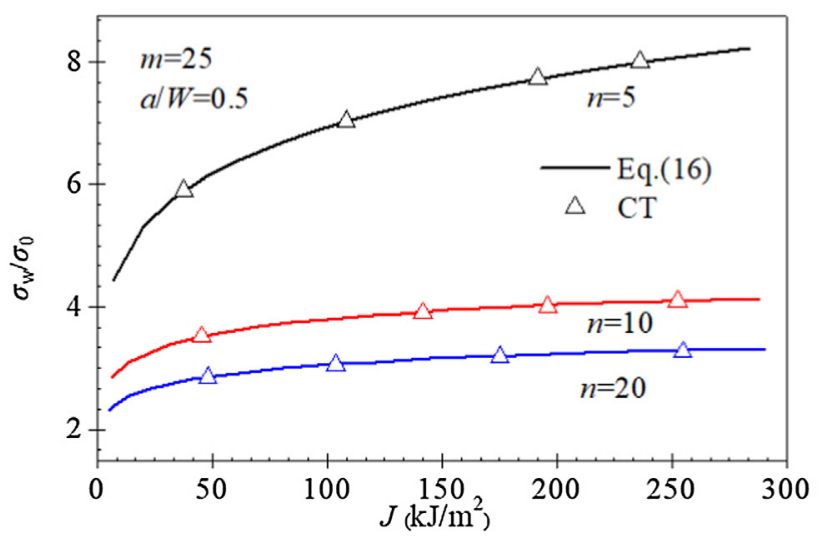

(f) CT with $a / W=0.5$ and $m=45$

Fig. 14. Verification of Weibull stress solution using specimen models for elastic plastic material including the effect of hardening exponents and Weibull modulus.

\subsubsection{Effect of Q-value}

Several cases are considered to investigate the influence of constraint parameter $Q$ on Weibull stress from -0.8 to 0.15 . The Weibull stress is computed by the proposed solution Eq. (16) with Weibull module $m=15$ for 3 types of elastic-plastic material, as shown in Fig. 15. Examination of these results leads to the following observations: (1) Weibull stress increases with $J$-integral. At low loading level, Weibull stress increases linearly with the increase of $J$-integral, but deviate from the line with further deformation due to the elastic plastic material model. In addition, the slope of the linear relation is roughly the same under low loading where the deformation satisfies small-scale yielding. This is coincident with the results described in Section 5.1.2 for elastic material, where $K$ still uniquely characterizes crack-tip conditions even though a plastic zone is present. (2) Weibull stress reduces with the decrease of $Q$ value under the same loading (same $J$-integral), especially for large loading. Meanwhile, fracture toughness decreases as constraint relaxes. Therefore, Weibull stress has been adopted to predict scale fracture toughness $[15,31]$. (3) The effect of constraint loss on Weibull stress is more obvious with the increase of material hardening exponent $n$. However, some new design of the structures inspired from composite materials made with the additive manufacturing technique may be helpful to reduce constraint loss $[32,33]$. 


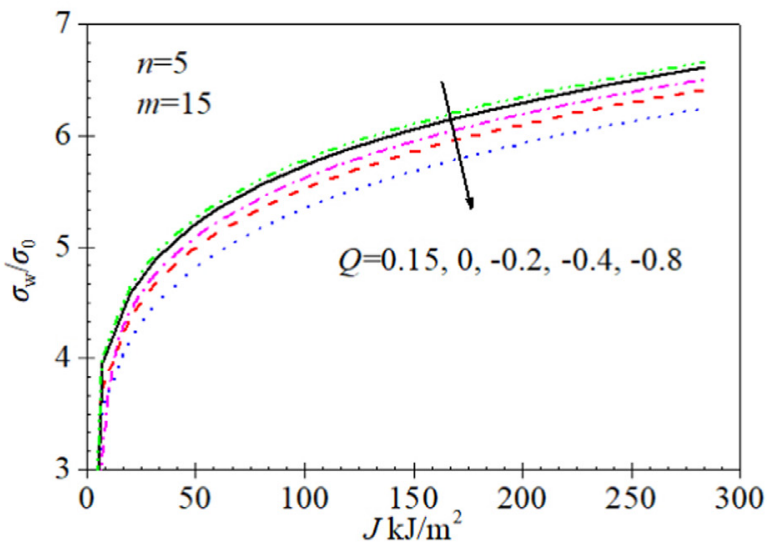

(a) $n=5$

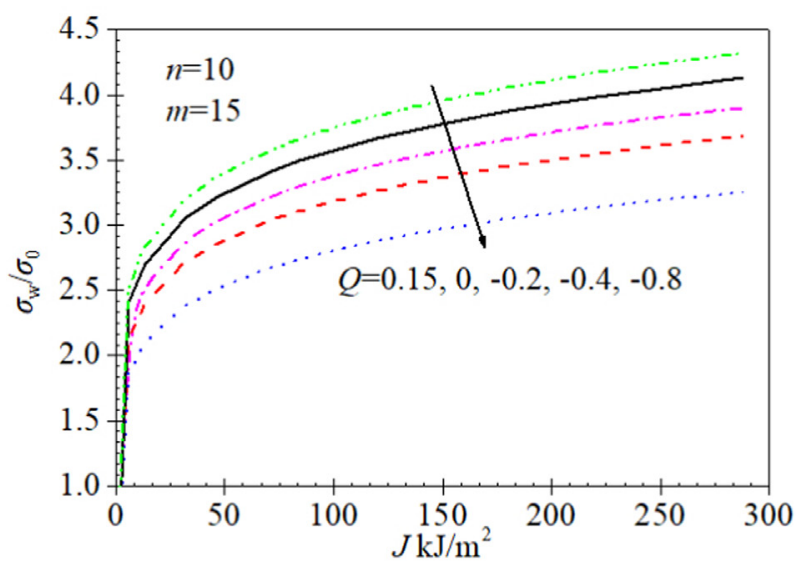

(b) $n=10$

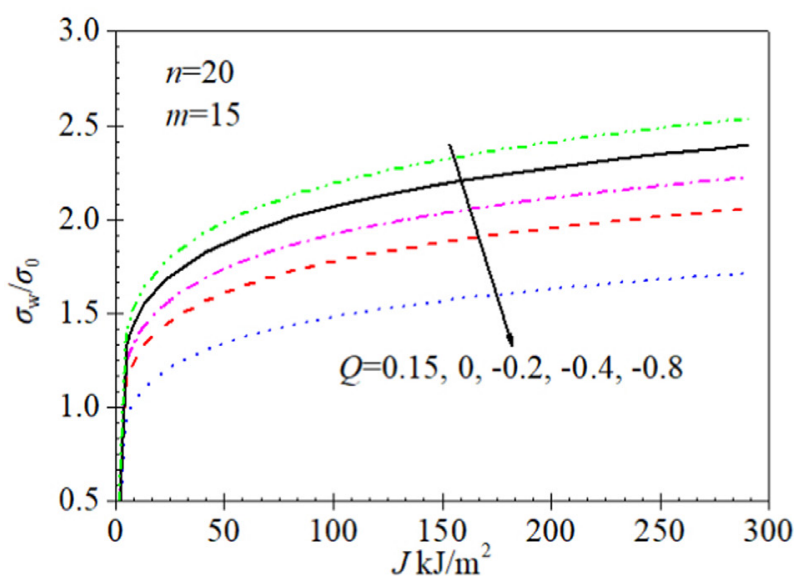

(c) $n=20$

Fig. 15. Weibull stress with $Q$ estimated by the proposed solution for elasticplastic material.

\section{Conclusions}

From a standpoint of fracture driving force, Weibull stress within the frame of local approach to fracture is solved with constraint effects characterized by $T$-stress for elastic material and $Q$ for elastic plastic material. These solutions are verified with existing solutions for $T=0$ and finite element solutions including modified boundary layer models, contact tension models and single-edge bend models. The constraint effect is discussed. The above work supports the following conclusions:
(1) For elastic material, Weibull stress has been derived and solved for notch in terms of $K$ and $T$-stress. The solution is coincident with the results by Lei et al. for zero $T$-stress and has been verified numerically using the results from finite element analyses. Good agreement has been obtained in all cases.

(2) $T$-stress has limited influence on the Weibull stress for a small Weibull modulus $m$, and the influence vanishes with the increased Weibull modulus. Weibull stress fails to interpret the constraint effect, where the elastic material model is assumed in the derivation of Weibull stress solution.

(3) For elastic plastic material, Weibull stress has been inferred and solved in terms of $J$-integral and $Q$. The coefficients of the solution are evaluated using finite element analysis with modified boundary layer models. The solution has been verified with the results from finite element analyses with specimen models and good agreement has been obtained.

(4) Weibull stress reduces with the decrease of $Q$ value, which is more obvious with the increase of material hardening exponent $n$. The Weibull stress solutions can be adopted to predict scale fracture toughness.

\section{Declaration of Competing Interest}

None.

\section{Acknowledgements}

The authors are grateful for the supports provided by the National Natural Science Foundation of China (51605435, 11602219, 11872364) and by CAS Hundred Talents Program.

\section{Appendix A. Supplementary material}

Supplementary data to this article can be found online at https:// doi.org/10.1016/j.tafmec.2019.102379.

\section{References}

[1] F.M. Beremin, A local criterion for cleavage fracture of a nuclear pressure vessel steel, Metall. Mater. Trans A. 14 (1983) 2277-2287, https://doi.org/10.1007/ BF02663302.

[2] F. Mudry, A local approach to cleavage fracture, Nucl. Eng. Des. 105 (1987) 65-76, https://doi.org/10.1016/0029-5493(87)90230-5.

[3] B.Z. Margolin, V.A. Shvetsova, A.G. Gulenko, et al., Prometey local approach to brittle fracture: development and application, Eng. Fract. Mech. 75 (11) (2008) 3483-3498, https://doi.org/10.1016/j.engfracmech.2007.05.002.

[4] G. Qian, W.S. Lei, M. Niffenegger, V.F. Gonzalez, On the temperature independence of statistical model parameters for cleavage fracture in ferritic steels, Philo. Mag. 98 (2018) 959-1004.

[5] G. Qian, W.S. Lei, L. Peng, Z. Yu, M. Niffenegger, Statistical assessment of notch toughness against cleavage fracture of ferritic steels, Fatigue Fract. Eng. Mater. Struct. 41 (2018)1120-1131.3.

[6] G. Qian, W. Lei, Z. Yu, F. Berto, Statistical size scaling of breakage strength of irregularly-shaped particles, Theo. App. Fract. Mech. 102 (2019) 51-58.

[7] G. Qian, W. Lei, A statistical model of fatigue failure incorporating effects of specimen size and load amplitude on fatigue life, Phil. Mag. (2019), https://doi.org/ 10.1080/14786435.2019.1609707.

[8] Y. Lei, N.P. O'Dowd, E.P. Busso, G.A. Webster, Weibull stress solutions for 2-D cracks in elastic and elastic-plastic materials, Int. J. Fract. 89 (1998) 245-268, https://doi.org/10.1023/A;10074359.

[9] N.P. O'Dowd, Y. Lei, E.P. Busso, Prediction of cleavage failure probabilities using the Weibull stress, Eng. Fract. Mech. 67 (2000) 87-100, https://doi.org/10.1016/ S0013-7944(00)00051-5.

[10] S.G. Larsson, A.J. Carlsson, Influence of non-singular stress terms and specimen geometry on small-scale yielding at crack tips in elastic plastic material, J. Mech. Phys. Solids 21 (4) (2009) 263-277, https://doi.org/10.1016/0022-5096(73) 90024-0.

[11] M. Gupta, R.C. Alderliesten, R. Benedictus, A review of T-stress and its effects in fracture mechanics, Eng. Fract. Mech. 134 (2015) 218-241, https://doi.org/10. 1016/j.engfracmech.2014.10.013.

[12] N.P. O'Dowd, C.F. Shih, Family of crack-tip fields characterized by a triaxiality parameter - I: structure of fields, J. Mech. Phys. Solids 39 (8) (1991) 989-1015.

[13] N.P. O'Dowd, C.F. Shih, Family of crack-tip fields characterized by a triaxiality parameter - II: fracture applications, J. Mech. Phys. Solids 40 (5) (1992) 939-963, 
https://doi.org/10.1016/0022-5096(92)90057-9.

[14] C. Ruggieri, R.G. Savioli, R.H. Dodds, An engineering methodology for constrain corrections of elastic-plastic fracture toughness - Part II: effects of specimen geometry and plastic strain on cleavage fracture predictions, Eng. Fract. Mech. 146 (2015) 185-209, https://doi.org/10.1016/j.engfracmech.2015.06.087.

[15] R.G. Savioli, C. Ruggieri, Experimental study on the cleavage fracture behavior of an ASTM A285 Grade C pressure vessel steel, J. Press. Vessel Technol. 137 (2014) 021206, , https://doi.org/10.1115/1.4028003.

[16] X. Gao, J.A. Joyce, C. Roe, An investigation of the loading rate dependence of the Weibull stress parameters, Eng. Fract. Mech. 75 (2008) 1451-1467, https://doi. org/10.1016/j.engfracmech.2007.07.007.

[17] G. Qian, M. Niffenegger, Investigation of constraint and warm prestressing effects by means of a local approach to fracture, Eng. Fract. Mech. 136 (2015) 26-37, https://doi.org/10.1016/j.engfracmech.2015.01.031.

[18] X. Gao, C. Ruggieri, R.H. Dodds, Calibration of Weibull stress parameters using fracture toughness data, Int. J. Fract. 92 (1998) 175-200, https://doi.org/10.1023/ A:1007521530191.

[19] X. Gao, R.H. Dodds Jr, An engineering approach to assess constraint effects on cleavage fracture toughness, Eng. Fract. Mech. 8 (2001) 263-283, https://doi.org/ 10.1016/S0013-7944(00)00102-8.

[20] G. Qian, Y. Cao, M. Niffenegger, Y.J. Chao, W. Wu, Comparison of constraint analyses with global and local approaches under uniaxial and biaxial loadings, Europ. J. Mech A/Solids 69 (2018) 135-146, https://doi.org/10.1016/j. euromechsol.2017. 12.006.

[21] X. Qian, R.H. Dodds, S. Yin, R. Bass, Cleavage fracture modeling of pressure vessels under transient thermo-mechanical loading, Eng. Fract. Mech. 75 (2008) 4167-4189, https://doi.org/10.1016/j.engfracmech.2008.03.011.

[22] C. Ruggieri, R.H. Dodds, An engineering methodology for constraint corrections of elastic-plastic fracture toughness - Part I: a review on probabilistic models and exploration of plastic strain effects, Eng. Fract. Mech. 134 (2015) 368-390, https:// doi.org/10.1016/j.engfracmech.2014.12.015.

[23] M.L. Williams, On the stress distribution at the base of a stationary crack, J. Appl. Mech. 24 (1957) 109-114.

[24] T.L. Sham, The determination of the elastic T-term using higher order weight functions, Int. J. Fract. 48 (1991) 81-102.

[25] A.H. Sherry, C.C. France, M.R. Goldthorpe, Compendium of T-stress solution for two and three dimensional cracked geometries, Fati Fract. Eng. Mater. Struct. 18 (1995) 141-155, https://doi.org/10.1111/j.1460-2695.1995.tb00148.x.

[27] J.R. Rice, Limitations to the small scale yielding approximation for crack tip plasticity, J. Mech. Phys. Solids 22 (1974) 17-26, https://doi.org/10.1016/00225096(74)90010-6.

[28] S. Cravero, C. Ruggieri, A two-parameter framework to describe effects of constraint loss on cleavage fracture and implications for failure assessments of cracked components, J. Braz. Mech. Sci. Eng. 4 (2003) 403-412, https://doi.org/10.1590/ S1678-58782003000400013.

[29] M. Moattari, I. Sattari-Far, Modification of fracture toughness master curve considering the crack-tip Q-constraint, Theoret. Appl. Fract. Mech. 90 (2017) 43-52, https://doi.org/10.1016/j.tafmec.2017.02.012.

[30] M. Nevalainen, R.H. Dodds, Numerical investigation of 3-D constraint effects on brittle fracture in SE(B) and C(T) specimens, Int. J. Fract. 74 (2) (1995) 131-161, https://doi.org/10.1007/BF00036262.

[31] Z. Zhang, X. Qian, Effect of experimental sample size on local Weibull assessment of cleavage fracture for steel, Fati. Fract. Eng. Mater. Struct. 40 (2017) 1128-1142, https://doi.org/10.1111/ffe.12571.

[32] Z. Wang, W. Wu, G. Qian, J. Oliveira Correia, L. Sun, In-situ SEM investigation on fatigue behaviors of additive manufactured Al-Si10-Mg alloy at elevated temperature, Eng. Fract. Mech. 214 (2019) 149-163.

[33] W. Wu, W. Hu, G. Qian, H. Liao, X. Xu, F. Berto, Mechanical design and multifunctional applications of chiral mechanical metamaterials: a review, Mater. Des. 18 (107950) (2019) 1-13. 